\title{
The Role of Fluoride Varnishes for Caries Control in Children from 3 to 6 Years of Age
}

\author{
Dr Dobrinka Damyanova Phd Student ${ }^{1}$, Dr Sirma Angelova Phd Student ${ }^{1}$, Dr \\ Teodora Targova-Dimitrova Phd ${ }^{2}$, Assoc. Prof. Dr Katerina Ivanova C.M.Sc. ${ }^{3}$ \\ ${ }^{l}$ assistant Professor,Medical University-Varna, Bulgaria, Faculty Of Dental Medicine, Department Of \\ Pediatric Dental Medicine \\ 2 assistant Professor,Medical University-Varna, Bulgaria, Faculty Of Dental Medicine, Department Of \\ Pariodotology And Dental Implantology \\ ${ }^{3}$ associate Professor,Medical University-Varna, Bulgaria, Faculty Of Dental Medicine, Department of \\ Pediatric Dental Medicine
}

\begin{abstract}
Background: Topical fluoride varnishes have been widely applied as a non-operative method of caries prevention for more than three decades. The aim of this study was to estimate the effectiveness of non-invasive treatment of dental caries as an item of a program for prevention of dental caries in deciduous teeth.

Material and Methods: We included 100 patients from 3 to 6 years of age in controlled clinical trials comparing the usage of fluorine varnish-Clinpro ${ }^{\mathrm{TM}}$ White Varnish with TCP (Tri-Calcium phosphate) (3M) applied for one year period. The control group of the same age included 100 children with no operative treatment or fluorine supplements application. The duration of the study was 12 months. Fluoride varnish was applied on carious lesions - dla and dlb, diagnosed with DIAGNOdent Pen, on smooth surfaces of temporary teeth. In statistical data processing is implemented parametric theory of assessment of statistical hypotheses by comparing the relative values of the two samples.

Results: For the experimental group the index of caries activity equals to 1.19\%. Epidemiology of dental caries in persons equals to $88 \%$ in the experimental group. Fluoride varnish clinical application reduces the level of decay risk on smooth tooth surfaces with $49.79 \%$ after a 12 months period of application.

Conclusion: Based on the facilities of advanced laser techniques we can assess the efficiency of fluorine varnishes application.
\end{abstract}

Keywords: non-operative method, fluoride varnish, deciduous teeth.

\section{Introduction}

Topical fluoride varnishes have been widely applied as a non-operative method of caries prevention for more than three decades $[1,2,3,4]$.

Nowadays scientific investigations have been related with new conceptions in the scope of cariesology $[5,6]$. Contemporary literature sources concern tooth decay as a disease with behavioural nature, which has been initiated long before the occurrence of a cavity lesion. Various approaches of prevention and treatment of tooth decay process at the stage before its progression, namely stabilization or reversibility of lesions, are characterizing the up-to-date tendencies in scientific researches and clinical practice [5, 6, 7]. Today are introduced innovative diagnostic scales for early diagnostic procedures. For recording of initial lesions an optimized complex of diagnostic criteria is implemented $[8,9,10,11]$. Caries risk assessment is performed on individual and local level $[6,7]$. Worldwide scientific literature accentuates on plenty of ascertainments for the reversibility of tooth decay lesions $[10,12,13,14]$. New and more efficient products for non-invasive treatment of caries and decay lesions arrest are being applied. The aim of this study was to estimate the effectiveness of noninvasive treatment of dental caries as an item of a program for prevention of dental caries in deciduous teeth. Tasks:

1. Investigation of caries activity in children from 3 to 6 years of age for a period of one year.

2. Investigation of caries reduction in children from 3 to 6 years of age for a period of one year after professional application of Clinpro TM White Varnish with TCP (Tri-Calcium phosphate) (3M)

\section{Material and Methods}

We included 100 patients of $3,4,5$ and 6 years of age in controlled clinical trials comparing the usage of fluorine varnish-Clinpro ${ }^{\mathrm{TM}}$ White Varnish with TCP (Tri-Calcium phosphate) (3M) applied during the first month every week and afterwards on an interval of 3 months for one year period. The control group of the same age included 100 children with no operative treatment or fluorine supplements application. The duration of the 
study was 12 months. Fluoride varnish was applied on carious lesions - d1a and d1b, diagnosed with DIAGNOdent Pen, on smooth surfaces of temporary teeth. In statistical data processing is implemented parametric theory of assessment of statistical hypotheses by comparing the relative values of the two samples.

\section{Results}

The index of caries activity for the control group amounts to $2.37 \%$. For the experimental group the index of caries activity equals to $1.19 \%$ (Figure 1 ).

Fig. 1. The index of caries activity on tooth surfaces of the temporary teeth for the control and test group ranges from $2.37 \%$ to $1.19 \%$

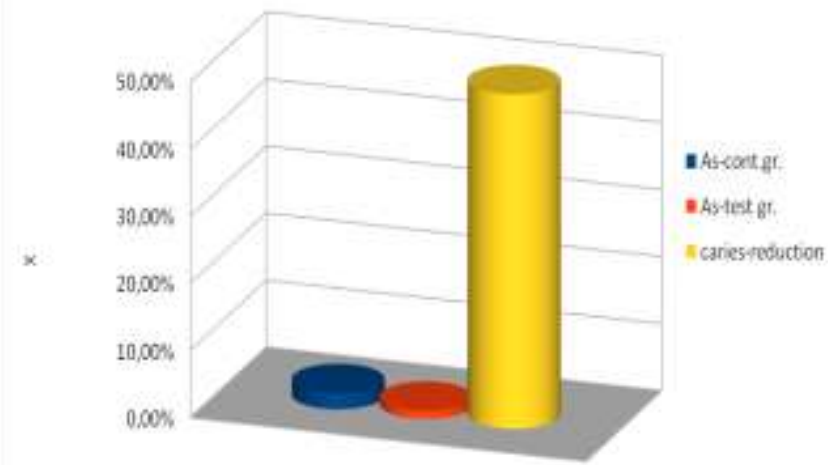

As-control group - index of caries activity on tooth surfaces in the control group $=2.37 \%$; As-test group - index of caries activity on tooth surfaces in the test group $=1.19 \%$; Caries-reduction - index of caries reduction for a period of one year $=49.79 \%$; Epidemiology of dental caries in persons equals to $88 \%$ in the experimental group (Figure 2).

Fig. 2. Epidemiology of dental caries in persons amounts to $88 \%$ in the test group. Principally, the index of caries reduction ranges from 5\% to $63 \%$. In our study it equals to $49.79 \%$

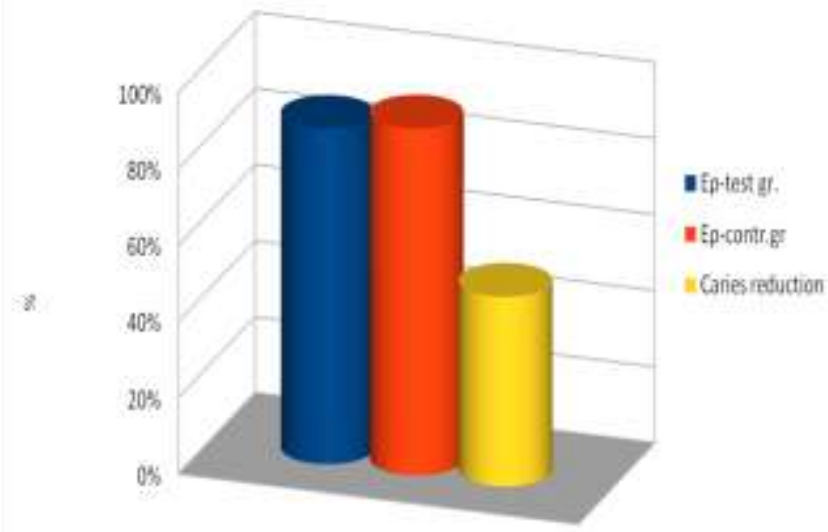

Ep - test group- epidemiology of tooth decay in persons-test group $=88 \%$; Ep - contr. groupepidemiology of tooth decay in persons-control group $=91 \%$; Caries-reduction - index of caries reduction for a period of one year $=49.79 \%$;

Fluoride varnish clinical application reduces the level of decay risk on smooth tooth surfaces with $49.79 \%$ after a 12 months period of application (Figures 3,4 ). 
Fig. 3. Values of epidemiology indices in children of different age of the test group

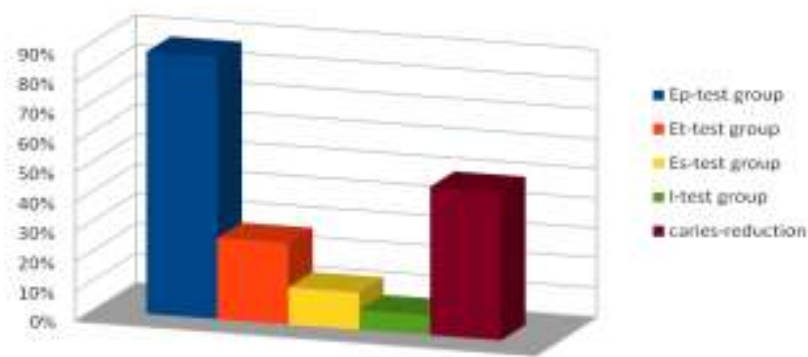

Ep - epidemiology of tooth decay in persons-test group $=88 \%$; Et - epidemiology of tooth decay in teeth-test group $=27.27 \%$; Es - epidemiology of tooth decay in surfaces-test group $=12.45 \%$; I - intensity of tooth decay in the test group $=6.56 \%$; Caries-reduction - index of caries reduction for a period of one year $=$ $49.79 \%$;

Fig. 4. Values of epidemiology indices in children of different age of the control group

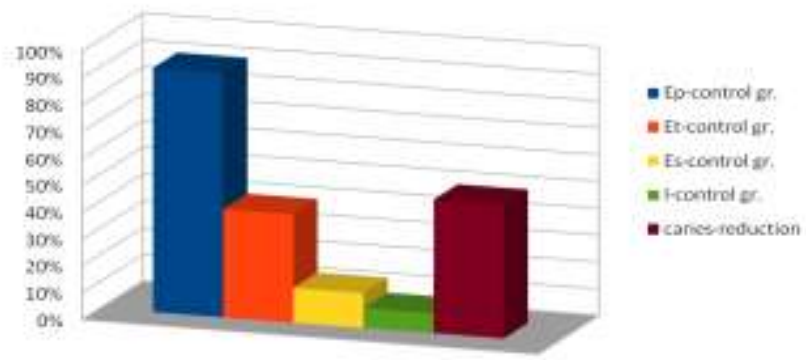

Ep - epidemiology of tooth decay in persons-control group $=91 \%$; Et - epidemiology of tooth decay in teeth-control group $=40.36 \%$; Es - epidemiology of tooth decay in surfaces-control group $=12.96 \%$; I intensity of tooth decay in the control group $=7.41 \%$; Caries-reduction - index of caries reduction for a period of one year $=49.79 \%$;

Comparative analysis of tooth decay of children studied in groups. When comparing with the experimental and control group of children on:

1."epidemic of dental caries in persons", shows that:

$t=0.69$, therefore $t<1.96$, this means that the difference is not significant $\Rightarrow>$ varnish is no reason for the difference in the outcome of the two studied groups of patients. With tooth decay threshold d1 is high in both treatment groups of children.

\section{2. epidemic of dental caries in teeth:}

$\mathrm{t}=1,97 \Rightarrow$ the difference between the experimental and control groups was significant. $\mathrm{t}=1.97$, therefore t> 1.96, this means that the difference is significant $\Rightarrow>$ Varnish is the reason for the difference in the studied experimental and control groups. This means that the initial lesions have undergone back development and are stationed in very early stages. This is due to the lower threshold of diagnosis and the success of preventive and non-invasive treatment of early caries lesions and d1a d1b with mineralized varnish CV.

3. "epidemic of dental caries on surfaces" shows that: $t=0,11 \Rightarrow$ difference is not significant.

Intensity of caries": $t=0,24=>$ difference is not significant. Not observed significant differences from the comparative analysis of the indices of caries statistics: epidemic of dental caries on surfaces and intensity of dental caries. 


\section{Discussion}

The proper health status of all the deciduous teeth in children of different ages is undoubtedly of great significance. This study confirms that the purpose of the non-invasive treatment approaches is feasible. Nowadays Dental Medicine specialists can improve the quality of prophylactic cares and treatment methods implemented for the control of tooth decay [9, 12]. More than 80 studies have been conducted with the application of these products (fluoride varnishes) and a meta-analysis of eight studies reported a reduction in dental caries amounting to $38 \%$ (Helfenstein \& Steiner, 1994) $[(8,9]$. It has been ascertained that delivery of high doses of fluorides of this type results in the local formation of calcium fluoride, which can act as a reservoir for the slow release of fluorides (Rolla \& Saxegard, 1990) [15]. An optimum fluoride-delivery system would be one that supplies small amounts of fluoride throughout the day so that consistent, elevated plaque fluoride levels are maintained with little or no individual effort required [7]. Essential is also the role of parents who are responsible for optimization of oral hygiene procedures performed by their children. Most important is the fact that the first step in arrest of further lesion progress is removal of the acid-maintaining factor of the disease, the caries-provoking plaque.

\section{Conclusions}

1. Based on the facilities of advanced laser techniques we can assess the efficiency of fluorine varnishes application.

2. Fluoride varnish clinical application reduces the level of decay risk on smooth tooth surfaces with $49.79 \%$ after a 12 months period of application.

3. Implementation of Clinpro TM White Varnish with TCP (Tri-Calcium phosphate) (3M) is efficient in preventing enamel demineralization as a method for non-invasive treatment of temporary teeth. One-time application in the beginning of treatment may not be enough to arrest process of demineralization affecting the sub-superficial enamel layer. In order to achieve significant preventive and therapeutic results varnish application is better conducted on every 6 months or more frequently-on each 3 months for a period of 1 year.

\section{References}

[1]. Divaris K, Preisser JS, Slade GD. Surface-Specific Efficacy of Fluoride varnish in caries prevention in the Primary dentition: Rezults of a Community Randomized clinical trial. Caries Res 2012 Nov 27, 47:78-87.

[2]. Peneva M. Caries in the XX century. Sofia: East-West, 2008:13-16;

[3]. Vutov M, Krumova Em, Ivanova K, Indjova K. Prevalence of dental caries in children and students from Plovdiv. Stom. (S.) 1983, $1: 1-8$.

[4]. www.Manufacturers/Products/Preventive-Dentistry/Dental-Prevention/Tooth-Desensitiser/.

[5]. Featherstone J. Dental caries: a dynamic disease process. Australian Dental Journal 2008 Aug.,18(53):286-291.

[6]. Featherstone John DB. The science and practice of caries prevention. JADA 2000 July, 131(7):887-898.

[7]. Fejerskov O, Kidd E. Dental caries, the disease and its clinical management. Bleckwell, Munksgaard 2004, IV 18:318.

[8]. Helfenstein U, Steiner M. Fluoride varnishes (Duraphat): a meta-analysis. Community Dent Oral Epidemiol 1994, 22 : 1-5.

[9]. Steiner M, Marthaler TM, Wiesner V, Menghini G. Caries incidence in schoolchildren in the canton of Glarus 9 years after the introduction of highly fluoridated table salt (250 mg F/kg). Schweiz Monatsschr Zahnmed 1986, 96: 688-99.

[10]. Lussi A, Francescut P. Performance of conventional and new methods for the detection of occlusal caries in deciduous teeth Caries Res 2003 Jan-Feb, 37,1:2-7.

[11]. Steiner M, Menghini G, Marthaler TM. The caries incidence in schoolchildren in the Canton of Glarus 13 years after the introduction of highly fluoridated salt. Schweiz Monatsschr Zahnmed 1989, 99:897-901.

[12]. De Benedetto MS, Morais CC, Novaes TF, de Almeira Rodrigues J, Brada MM, Mendes FM. Comparing the reliability of a new fluorescence camera with conventional laser fluorescence devices in detecting caries le-sions in occlusal and smooth surfaces of primary teeth. Laser Med Sci. 2011 Mar, 26(2):157-162.

[13]. Mitropoulos P. et al. Diagnostic performance of the visual caries classification system ICDAS II versus radiography and microcomputed tomography for proximal caries detection; An in vitro study. J Dent 2010 Nov, 38(11):859-67.

[14]. Pitts NB, Stamm J. International Consensus Workshop on Caries Clinical Trials (ICW-CCT) Final Consensus Statements: Agreeing Where the Evidence Leads. J Dent Res 2004:83.

[15]. Rolla G, Saxegaard E. Critical evaluation of the composition and use of topical fluorides, with emphasis on the role of calcium fluoride in caries inhibition. J Dent Res 1990, 69: 780-5. 